\title{
Assistência de enfermagem domiciliar ao portador de hanseníase: Um incentivo ao autocuidado
}

\author{
Home nursing care for leprosy patients: An incentive to self-care
}

Cuidado de enfermería a domicilio para pacientes con leprosa: Un incentivo para el autocuidado

Allan Bruno Alves de Sousa Santos ORCID: https://orcid.org/0000-0001-6412-7164

Faculdade de Educação São Francisco, Brasil

E-mail: abass@faesf.com.br

Suelen Visniewski Barbosa

ORCID: https://orcid.org/0000-0001-8111-1236 Universidade Federal de pelotas, Brasil

E-mail: suelenbarbosa1@ hotmail.com

Kallyne dos Santos

ORCID: https://orcid.org/00000002-1938-0539 Centro Universitário Integrado, Brasil

E-mail: kallynesant@outlook.com

Leandro Barbosa Teixeira

ORCID: https://orcid.org/00000002-9444-7828

Universidade Estácio de Sá, Brasil

E-mail: Lbtteixeira@hotmail.com

Cássia Isabelle Carvalho Alves Moreira

ORCID: https://orcid.org/00000002-0224-4629

Christus Faculdade do Piauí, Brasil

E-mail: viksilva20.cm@gmail.com

Mateus Silva Soares

ORCID: https://orcid.org/0000-0002-5562-4908

Faculdade de Educação São Francisco, Brasil E-mail:mateussilva8945@gmail.com

Jackson Cabral Oliveira

ORCID: https://orcid.org/00000002-7200-8103

Faculdade de Educação São Francisco, Brasil

E-mail: jacksoncabraloliveira@gmail.com

Mikaelle Alves Silva

ORCID: https://orcid.org/0000-0002-9336-1491

Universidade Federal do Espírito Santo, Brasil E-mail: farm.mikaelle@gmail.com

Carla Conrado Barbosa Leite

ORCID: https://orcid.org/00000002-7033-3682

Faculdade da Polícia Militar, Brasil

E-mail: carlaconradobl@gmail.com

Amanda Costa Maciel

ORCID: https://orcid.org/00000002-2668-037X

Universidade Federal de Sergipe, Brasil

E-mail: amandacmaciel@hotmail.com

Sara Paulino dos Anjos

ORCID: https://orcid.org/00000002-9568-8666

Universidade Paulista, Brasil

E-mail: sarapaulinodosanjos@hotmail.com

Dalton Ferreira Matos

ORCID: https://orcid.org/0000-0002-6188-7536

Universidade Federal de Sergipe, Brasil

E-mail: daltonmatosgn@gmail.com

Nair Arrais Leite

ORCID: https://orcid.org/0000-0003-2773-9350

Faculdade de Educação São Francisco, Brasil

E-mail: nairarrais@outlook.com

Débora de Oliveira Lima

ORCID: https://orcid.org/0000-0002-8706-9451

Universidade Federal do Piauí, Brasil

E-mail: deboralima209@gmail.com 


\begin{abstract}
Resumo
Este estudo tem como objetivo identificar na literatura as principais ações desenvolvidas pela equipe de enfermagem no âmbito domiciliar, para ofertar qualidade de vida e melhorar o prognóstico da pessoa portadora de hanseníase, apontando a importância de praticar o autocuidado. Trata-se um uma revisão do tipo descritiva de caráter qualitativo, a coleta de dados ocorreu nas bases: LILACS; BDENF e ScieLO. Foi utilizado os descritores "Assistência de Enfermagem Domiciliar; Hanseníase; Autocuidado" localizados na lista dos Descritores em Ciências da Saúde (DECs), e ocorreu no período de junho a julho de 2021. Foram identificados na primeira etapa da pesquisa um quantitativo de 107 estudos, que após a aplicação dos critérios de inclusão e exclusão, concluiu-se como amostra final um quantitativo de 7 estudos usados na pesquisa. A principal dificuldade estabelecida para o cuidado efetivo no domicílio foi a falta de preparo da equipe de saúde e a desinformação por parte do paciente portador de hanseníase. Além disso, aponta-se também a falta de adesão ao sistema PQT, como um dos principais fatores de risco desencadeante de complicações. Portanto evidenciou-se a necessidade da promoção de saúde em todos os eixos do cuidado, em especial praticas voltadas a atenção domiciliar, e a capacitação da equipe de saúde para exercer as atividades no domicílio com efetividade.
\end{abstract}

Palavras-chave: Assistência de enfermagem domiciliar; Hanseníase; Autocuidado.

\begin{abstract}
This study aims to identify in the literature the main actions developed by the nursing team at home, to offer quality of life and improve the prognosis of people with leprosy, pointing out the importance of practicing self-care. This is a descriptive review of qualitative character, data collection occurred in the following databases: LILACS; BDENF and ScieLO. The descriptors "Home Nursing Care; Leprosy; Self-care" located in the list of Health Sciences Descriptors (DECs), and took place from June to July 2021. In the first stage of the research, 107 studies were identified, which after applying the inclusion and exclusion criteria, the final sample was concluded with a quantitative of 7 studies used in the research. The main difficulty established for effective home care was the lack of preparation of the health team and lack of information on the part of the leprosy patient. In addition, the lack of adherence to the MDT system is also pointed out as one of the main risk factors triggering complications. Therefore, the need for health promotion in all axes of care was evidenced, especially practices aimed at home care, and the training of the health team to carry out activities at home effectively.
\end{abstract}

Keywords: Home nursing assistance; Leprosy; Self-care.

\title{
Resumen
}

Este estudio tiene como objetivo identificar en la literatura las principales acciones que realiza el equipo de enfermería en el hogar, para ofrecer calidad de vida y mejorar el pronóstico de las personas con lepra, señalando la importancia de practicar el autocuidado. Se trata de una revisión descriptiva de carácter cualitativo, la recolección de datos se realizó en las siguientes bases de datos: LILACS; BDENF y ScieLO. Los descriptores "Atención de enfermería domiciliaria; Lepra; Autocuidado" ubicado en la lista de Descriptores de Ciencias de la Salud (DECs), y se llevó a cabo de junio a julio de 2021. En la primera etapa de la investigación se identificaron 107 estudios, que luego de aplicar los criterios de inclusión y exclusión, la muestra final se concluyó con un análisis cuantitativo de 7 estudios utilizados en la investigación La principal dificultad establecida para una atención domiciliaria eficaz fue la falta de preparación del equipo de salud y la falta de información por parte del enfermo de lepra. Además, la falta de adherencia al sistema MDT también se señala como uno de los principales factores de riesgo desencadenantes de complicaciones. Por tanto, se evidenció la necesidad de la promoción de la salud en todos los ejes del cuidado, especialmente las prácticas dirigidas al cuidado domiciliario, y la formación del equipo de salud para realizar las actividades en el hogar de forma eficaz.

Palabras clave: Asistencia de enfermería a domicilio; Lepra; Autocuidado.

\section{Introdução}

A hanseníase continua sendo um importante problema para saúde da população, no contexto global, porém ainda sofre grandes taxas de negligencia, quando interpretada de forma erronia, na qual não atribuem sua verdadeira importância (Tiwari \& Richardus, 2016).

Conforme as estatísticas da Organização Mundial da Saúde (OMS), são apontados mais de 200 mil casos por ano de hanseníase, sendo uma porcentagem significativas desses casos, atribuídos a portadores que servem apenas de reservatório, e 
assim disseminam a doença livremente. Reconhecendo que o sistema de saúde apresenta falhas, no combate à doença, podendo ser apontado: A falta de uma captação eficiente na fase inicial da patologia, o que contribui para altas taxas de deformidades (Estratégia global para a hanseníase, 2016).

O Brasil no ano de 2018, registrou cerca de 27 mil casos de hanseníase, e isso o caracteriza como segundo pais do mundo na listagem de novos casos, ficando atras somente da índia. No panorama nacional, os casos em território brasileiro correspondem a cerca de $93 \%$ de toda a ocorrência da doença em toda a américa. As regiões norte e Nordeste assumem o quantitativo de novos casos no país (Ministério da saúde,2017).

Segundo o Ministério da Saúde (2017), a Hanseníase é uma patologia crônica e infectocontagiosa, que acomete em preferencial os nervos da superfície do tegumento, e é dada a seguinte classificação: Indeterminada, tuberculóide, diforma e virchowiana.

O número de infectados se dá na maioria das vezes pelo convívio com pessoas que não foram submetidas ao tratamento, sendo no contexto familiar o principal foco contaminante, e vale ressaltar que grande parte da população é imune a bactéria Mycobacterium leprae (Ministério da saúde,2017).

Segundo Pinheiro et al (2014), as ações educativas realizadas na atenção domiciliar, busca através de um colhimento e prestação de serviços, fornecer uma melhor qualidade de vida aos portadores de hanseníase, e principalmente estimulam o autocuidado, o que se caracteriza fator importante na prevenção de deformidades. Ação fundamentada na teoria de Orem, na qual enfatiza a importância da pratica do autocuidado, ou seja, que cada paciente possui habilidades e competência para cuidar de si, e com acompanhamento diário da equipe de enfermagem (Sousa, Brito \& Silva,2012).

Com base na questão norteadora do estudo, tem-se o seguinte objetivo: Identificar na literatura as principais ações desenvolvidas pela equipe de enfermagem no âmbito domiciliar, para ofertar qualidade de vida e melhorar o prognóstico da pessoa portadora de hanseníase, apontando a importância de praticar o autocuidado.

\section{Metodologia}

Trata-se de um estudo do tipo revisão integrativa de aspecto qualitativo. Segundo Ercole, Melo \& Alcoforado (2014), esse tipo de revisão é utilizada para sintetização de resultados obtidos através de uma determinada pesquisa nas bases de dados, sobre uma temática especifica ou pergunta norteadora, de aspecto abrangente e sistemático.

Foi seguida as seguintes etapas para realização da pesquisa: 1) Elaboração da temática e pergunta norteadora através da estratégia PIco; Elaboração dos descritores no site DECs; Busca nas bases de dados selecionadas; Elaboração dos critérios de inclusão e exclusão; Analise crítica dos estudos incluídos na pesquisa; Apresentação e discussão dos resultados.

Para nortear o estudo, foi estabelecida a seguinte pergunta "O que a literatura aborda sobre a atuação da enfermagem no cuidado ao paciente portador de hanseníase no contexto domiciliar?".

Para o desenvolvimento da pesquisa, a busca foi realizada na Literatura Latino -Americana e do Caribe em Ciências da Saúde (LILACS); Scientific Electronic Library Online (SciELO); base de Dados de Enfermagem (BDENF). Foram utilizados os descritores "Assistência de Enfermagem Domiciliar; Hanseníase; Autocuidado", estabelecidos no site Descritores em Ciências da Saúde (DECs), e cruzados com o operador booleano" AND". A coleta foi realizada no período de junho a julho de 2021.

Foram incluídos no estudo artigos completos disponíveis na integra, que responderam ao objetivo do estudo nos idiomas português e inglês. Excluiu-se artigos incompletos, resumos, teses, dissertações, estudos com recorte temporal inferior a 2015. 


\section{Resultados e Discussão}

No fluxograma a seguir apresenta com detalhes toda a trajetória da pesquisa, apontando os critérios de seleção dos manuscritos.

Figura 1. Fluxograma com a trajetória da seleção dos manuscritos.

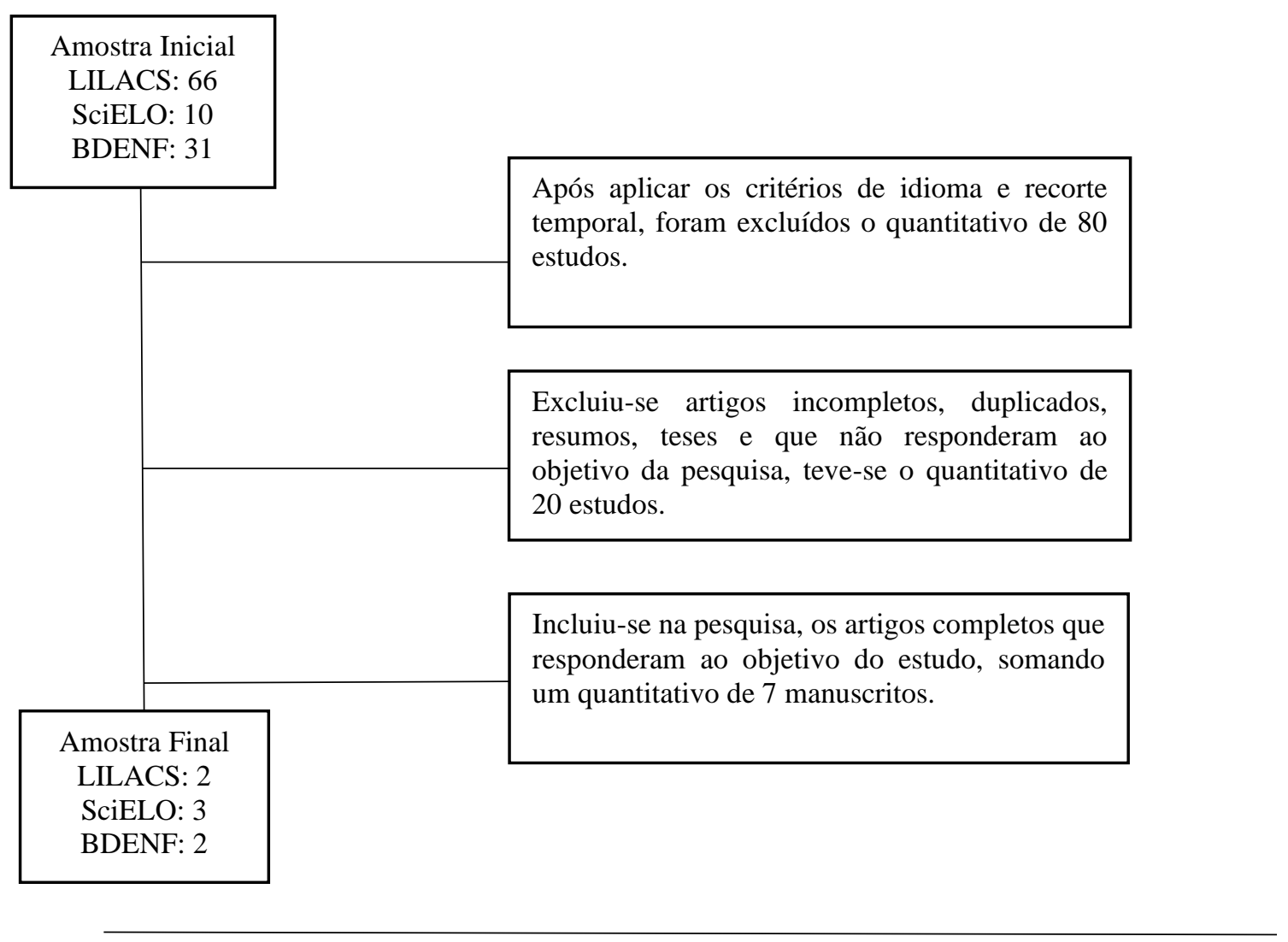

Fonte: Autores (2021).

No Fluxograma 1, consta-se a trajetória da pesquisa, que inicia a partir da coleta de dados nas bases de dados de forma ampla, na qual localizaram-se 107 manuscritos, que foram submetidos a primeira aplicação de critérios de elegibilidade, e assim observa-se uma amostra de 27 estudos. Foram excluídos artigos duplicados, incompletos e que não apresentavam similaridade com a pergunta norteadora do estudo, restando ao final das etapas de seleção, um número de 7 manuscritos.

Foi realizado a leitura dos artigos na integra, e constatou-se que os 7 respondiam à questão norteadora do estudo.

No Quadro 1, a seguir, detalha os artigos usados na pesquisa, segundo o autor, título, método e ano. 
Quadro 1 - Estudos tocantes a pesquisa.

\begin{tabular}{|c|c|c|c|}
\hline Autor & \begin{tabular}{|c|} 
Título \\
\end{tabular} & Método & Ano \\
\hline Souza et al. & $\begin{array}{l}\text { Autocuidado em hanseníase sob a ótica de grupos } \\
\text { operativos: uma abordagem qualitativa }\end{array}$ & $\begin{array}{l}\text { Estudo qualitativo do } \\
\text { tipo explicativo }\end{array}$ & 2021 \\
\hline Oliveira et al. & $\begin{array}{l}\text { Diagnósticos de enfermagem em pessoas } \\
\text { com hanseníase: aproximação entre teoria de orem e a } \\
\text { CIPE. }\end{array}$ & Estudo transversal descritivo. & 2020 \\
\hline $\begin{array}{l}\text { Lima, Barbosa, } \\
\text { Santos, } \\
\text { Nascimento, \& } \\
\text { D'azevedo. }\end{array}$ & Práticas de autocuidado em hanseníase: face, mãos e pés & Estudo qualitativo. & 2018 \\
\hline $\begin{array}{l}\text { Santos } \quad \& \\
\text { Ignotti. }\end{array}$ & $\begin{array}{l}\text { Prevenção de incapacidade física por hanseníase no Brasil: } \\
\text { análise histórica }\end{array}$ & Estudo documental. & 2018 \\
\hline Moura et al. & $\begin{array}{l}\text { Relação entre a Classificação Internacional de } \\
\text { Funcionalidade, Incapacidade e Saúde (CIF) e a limitação } \\
\text { de atividades e restrição à participação de indivíduos com } \\
\text { hanseníase. }\end{array}$ & \begin{tabular}{ll} 
Estudo & \multicolumn{2}{c}{ observacional } \\
transversal & analítico $\quad \mathrm{e}$ \\
descritivo &
\end{tabular} & 2017 \\
\hline $\begin{array}{l}\text { Sousa, Silva, \& } \\
\text { Xavier. }\end{array}$ & $\begin{array}{l}\text { Atributos da atenção primária em saúde no controle da } \\
\text { hanseníase: ótica do enfermeiro. }\end{array}$ & Estudo avaliativo. & 2017 \\
\hline $\begin{array}{l}\text { Ribeiro, } \\
\text { Castillo, Silva, } \\
\text { \& Oliveira. }\end{array}$ & $\begin{array}{l}\text { A visão do profissional enfermeiro sobre o tratamento da } \\
\text { hanseníase na atenção básica }\end{array}$ & Estudo descritivo qualitativo. & 2017 \\
\hline
\end{tabular}

Fonte: Autores (2021).

No Quadro 2, a seguir, apresenta os artigos usados no estudo, segundo sua autoria e objetivo.

Quadro 2 - Estudos tocantes a pesquisa.

\begin{tabular}{l|l}
\hline Autor & objetivo \\
\hline Souza et al. & $\begin{array}{l}\text { Analisar um grupo de apoio ao autocuidado para pessoas acometidas pela hanseníase } \\
\text { sob a ótica de grupos operativos. }\end{array}$ \\
\hline Oliveira et al. & $\begin{array}{l}\text { Analisar o perfil de diagnósticos de enfermagem em pessoas com hanseníase, } \\
\text { utilizando a teoria de orem e a Classificação Internacional para a Prática de } \\
\text { Enfermagem (CIPE®) }\end{array}$ \\
\hline Lima et al. & $\begin{array}{l}\text { Analisar as práticas de autocuidado em face, mãos e pés realizadas por pessoas } \\
\text { atingidas pela hanseníase. }\end{array}$ \\
\hline $\begin{array}{l}\text { Santos \& } \\
\text { Ignotti. }\end{array}$ & $\begin{array}{l}\text { Descrever as mudanças, de forma cronológica, nas orientações técnicas para } \\
\text { prevenção das incapacidades físicas por hanseníase no Brasil. }\end{array}$ \\
\hline Moura et al. & $\begin{array}{l}\text { Relacionar a CIF com a limitação de atividade e restrição à participação de } \\
\text { indivíduos com hanseníase, aferidas por meio da escala SALSA. }\end{array}$ \\
\hline $\begin{array}{l}\text { Sousa; Silva } \\
\& \text { Xavier. }\end{array}$ & $\begin{array}{l}\text { Avaliar a presença e a extensão dos atributos essenciais e derivados da atenção } \\
\text { primária em saúde no programa de controle da hanseníase sob a ótica do enfermeiro. }\end{array}$ \\
\hline Ribeiro et al. & $\begin{array}{l}\text { Avaliar a visão dos enfermeiros atuantes na Atenção Básica (AB) sobre o tratamento } \\
\text { da hanseníase. }\end{array}$ \\
\hline
\end{tabular}

Fonte: Autores (2021).

O ministério da saúde preconiza cartilhas e folhetos, estabelecendo a importância do autocuidado para o prognostico positivo do paciente hanseniano, e são disponibilizados nas unidades de saúde, em especial a atenção primaria, considerada a porta de entrada para o atendimento a pessoa portadora de hanseníase. Os profissionais promotores destes materiais educativos, devem ser submetidos a um constante processo de capacitação, para abordarem de forma ampla essa patologia e desenvolverem ações em todos os eixos do cuidado, desde o diagnostico há atividades educativas no domicilio para evitar deformidades (Lima et al,2018). 
Segundo os estudos obtidos por Sousa, Silva e Xavier (2017), o paciente acometido pela hanseníase, procura como atendimento inicial de saúde a APS, e nesse contexto ocorre o atendimento feito pela equipe multiprofissional, em essencial as ações de enfermagem, e assim caracteriza também, o cuidado continuo ou vinculo de longitudinalidade, o que corrobora com a ação desenvolvida no domicilio.

A orientação a família e comunidade, é uma das responsabilidades dos profissionais da APS, caracterizando-se como fator incentivador do autocuidado no domicilio, facilitando assim o planejamento e desenvolvimento de ações preventivas estabelecidas pelo próprio usuário (Sousa et al, 2017).

Para o desenvolvimento efetivo das ações domiciliares no autocuidado ao paciente hanseniano, é importante as ações educativas focadas no tratamento medicamentoso instituído na Atenção Primaria a Saúde. Em conformidade a estudos realizados por Ribeiro et al, foi evidenciado em seus resultados que a poliquimioterapia (PQT), é efetivo no tratamento da hanseníase, e quando seguido corretamente proporciona a cura da doença. O PQT foi instituído na APS nos anos de 1991, e é um dos principais responsáveis pela queda nas altas taxas da doença. Porém, mesmo com as orientações corretas, o tratamento ainda é abandonado por um pequeno número de pacientes, e sendo a ação da enfermagem e a promoção de conhecimentos a principal atividades para combater esse abandono (Ribeiro et al, 2017).

Os cuidados no domicilio se caracterizam importante atividade no tratamento da pessoa portadora de hanseníase. A educação em saúde, promovendo ações fáceis de executar, porém com uma ação terapêutica efetiva na prevenção de deformidades, sendo a enfermagem a orientadora de cuidados com a face, pele, mãos e pés, e sanando todas as dúvidas que o paciente possa apresentar. Enfatizando os resultados obtidos por Lima et al (2017), constatou-se que os pacientes apresentam continuamente o sentimento de medo, em relação as exacerbações da patologia, sendo comum a visita a Unidade básica de saúde, por pigmentação avermelhada na pele, característica principal dos efeitos colaterais do PQT.

Segundo os estudos de Santos e Ignotti (2018), no contexto domiciliar o paciente vai praticar e vivenciar as orientações de enfermagem para o autocuidado, podendo citar: a autoavaliação diariamente, cuidados de limpeza e inspeção no nariz, olhos, mãos e pés, executar exercícios de alongamento das mãos e pés, objetivando evitar o atrofiamento dos membros, e cuidados com objetos que são fatores de risco para queimaduras e lacerações. E em conformidade com os estudos de Moura $e t$ al (2017), onde foi avaliado 69 pacientes submetidos a Screening of Activity Limitation and Safety Awareness, conhecida como escala de salsa, na qual objetiva avaliar as limitações físicas do paciente, a assim, apontou-se que grande parte dos portadores de hanseníase não possuem informações sobre os riscos contidos no domicilio que podem desencadear uma deformidade no organismo, e assim é necessário que as orientações de autocuidado sejam intensificadas pela enfermagem em seu contexto familiar.

Dentre todas as dificuldades encontradas no domicilio, a não adesão ao regime medicamentoso é que se encontra com maior frequência, e por conseguinte obteve-se também os problemas relacionados a percepção sensorial, relacionada principalmente ao acometimento dos nervos periféricos, sendo a assistência de enfermagem o cuidado que busca muito além de identificar as principais causas de complicações, mais também fatores que atual indiretamente com o prognóstico positivo, enfatizando principalmente hábitos saudáveis de vida (Oliveira et al,2020).

O preconceito com pacientes hansenianos, favorece a exclusão social, o que as vezes deixa mais sequelas que a própria patologia, o que é aconselhado é a conversa em grupos de apoio, o que remete a uma educação continuada e trocas de experiencias em especial a práticas de autocuidado no domicílio (Souza et al,2021).

Um dos limites desse estudo, foi a pouca quantidade de artigos na integra, que abordassem com objetividade as ações da enfermagem desenvolvidas no contexto familiar, enfatizando o autocuidado a pessoas portadoras de hanseníase, e assim foi necessário a elaboração de novos estudos que abordem enfaticamente a ação desses profissionais no contexto do domicilio. 


\section{Conclusão}

O estudo é efetivo à medida que possibilita conhecer a ação da enfermagem no contexto domiciliar, enfatizando as atividades de autocuidado e a necessidade da educação em saúde, para o prognostico positivo do paciente. Com o conhecimento adquirido na revisão integrativa, observa-se todas as dificuldades que são impostas a assistência no domicilio e enfatiza a participação da APS na educação dos portadores de hanseníase.

Envidem-se a necessidade da promoção de saúde em todos os eixos do cuidado, em especial praticas voltadas a atenção domiciliar, e a capacitação da equipe de saúde para exercer as atividades no domicílio com efetividade.

Frente a problemática apontada no referente estudo, aponta-se a necessidade do desenvolvimento de novos estudos voltados a temática da assistência de enfermagem ao paciente hanseniano no domicilio.

\section{Referências}

Dayanne Alves Ribeiro, M., da Silva Castillo, I., Carlos Araujo Silva, J., \& Brito Oliveira, S. (2017). A visão do profissional enfermeiro sobre o tratamento da hanseníase na atenção básica. Revista Brasileira Em Promoção Da Saúde, 221-228. 10.5020/18061230.2017.p221

Ercole, F., Melo, L., \& Alcoforado, C. (2014). Integrative review versus systematic review. Reme: Revista Mineira De Enfermagem, 18(1). 10.5935/14152762.20140001

Ferreira de Oliveira, B., Arouca da Silva, M., Da Silva Carvalho, A., Silva, R., Da Silva Melo, A., \& Farias Cordeiro, C. et al. (2020). Diagnósticos, intervenções e resultados de enfermagem cipe ${ }^{\circledR}$ a uma paciente com pielonefrite: relato de caso. Revista Eletrônica Acervo Enfermagem, 1, e2900. 10.25248/reaenf.e2900.2019

Lima, M., Barbosa, F., Santos, D., Nascimento, R., \& D’Azevedo, S. (2018). Práticas de autocuidado em hanseníase: face, mãos e pés. Revista Gaúcha De Enfermagem, 39(0). 10.1590/1983-1447.2018.20180045

Lima de Sousa, R., Ribeiro de Lima Brito, R., \& Socorro Santa Brígida da Silva, Z. (2021). Dificuldades Encontradas Pelos Enfermeiros (As) Das Ubs De Uma Cidade Do Tocantins Frente À Prevenção De Incapacidades Em Hanseníase. Revista Científica Do ITPAC, 4(4). https://assets.unitpac.com.br/arquivos/Revista/54/5.pdf

Moura, E., Araújo, A., Silva, M., Cardoso, B., Holanda, M., Conceição, A., \& Dias, G. (2017). Relação entre a Classificação Internacional de Funcionalidade, Incapacidade e Saúde (CIF) e a limitação de atividades e restrição à participação de indivíduos com hanseníase. Cadernos Saúde Coletiva, 25(3), 355-361. $10.1590 / 1414-462 \times 201700030336$

Madelon Nascimento Souza, N., de Lima Belmonte, M., Geórgia Torres Alves, M., Delmondes do Nascimento, R., Farias Gomes, M., \& Christine Moura dos Santos, D. (2021). Autocuidado em hanseníase sob a ótica de grupos operativos: uma abordagem qualitativa. Online Braz J Nurs, 20(64). http://doi.org/10.17665/1676-4285.20216448

Ministério da Saúde, Secretaria de Vigilância em Saúde, Departamento de Vigilância das Doenças Transmissíveis. (2021). Guia prático sobre a hanseníase (2nd ed.). Brasília.

Pinheiro, M., Silva, S., Silva, F., Ataide, C., Lima, I., \& Simpson, C. (2014). Knowledge On Prevention Of Disabilities In A Hanseniasis Self-Care Group. REME: Revista Mineira De Enfermagem, 18(4). 10.5935/1415-2762.20140066

Regional Office for South-East Asia, W. (2021). Global Leprosy Strategy 2016-2020. Accelerating towards a leprosy-free world. Monitoring and Evaluation Guide. https://apps.who.int/iris/handle/10665/254907

Santos, A., \& Ignotti, E. (2020). Prevenção de incapacidade física por hanseníase no Brasil: análise histórica. Ciência \& Saúde Coletiva, 25(10), 3731-3744. $10.1590 / 1413-812320202510.30262018$

Sousa, G., Silva, R., \& Xavier, M. (2017). Atributos Da Atenção Primária Em Saúde No Controle Da Hanseníase: Ótica Do Enfermeiro. Revista Baiana De Enfermagem $31,(1) .10 .18471 /$ rbe.v31i1.17251

Tiwari, A., \& Richardus, J. H. (2016). Investment case concepts in leprosy elimination: A systematic review. Leprosy review, 87(1), 2-22. 